\title{
Gradhiva
}

GRADHIV

Revue d'anthropologie et d'histoire des arts

$21 \mid 2015$

Création plastique d'Haïti

\section{Loin de Django, la musique des Manouches de Pau}

Far away from Django: the music of the Gypsies in Pau

Jean-Luc Poueyto

\section{OpenEdition}

Journals

Édition électronique

URL : http://journals.openedition.org/gradhiva/2977

DOI : 10.4000/gradhiva. 2977

ISSN : 1760-849X

Éditeur

Musée du quai Branly Jacques Chirac

Édition imprimée

Date de publication : 1 février 2015

Pagination : 182-205

ISBN : 978-2-35744-075-3

ISSN : 0764-8928

Référence électronique

Jean-Luc Poueyto, «Loin de Django, la musique des Manouches de Pau », Gradhiva [En ligne],

21 | 2015, mis en ligne le 01 février 2015, consulté le 19 avril 2019. URL : http://

journals.openedition.org/gradhiva/2977; DOI : 10.4000/gradhiva.2977

(c) musée du quai Branly 


\section{Loin de Django, la musique des Manouches de Pau}

par Jean-Luc Poueyto

Contrairement à ce que l'on pourrait croire, les Manouches de la région paloise ne jouent pas de «jazz manouche». Bien au contraire, en famille, on joue de l'opéra italien, de la musique classique romantique, de la bossa nova, de la chanson de variété française et internationale des années 1960 et 1970. Un tel répertoire s'inscrit dans l'histoire de ces familles, depuis leurs séjours en Espagne durant la première partie du $x x^{\mathrm{e}}$ siècle jusqu'à leur installation à Marseille à partir des années 1940, puis à Pau il y a une cinquantaine d'années. Nous retrouvons ici, comme dans la plupart des pratiques culturelles observées chez les Manouches, un processus d'appropriation qui consiste à donner un sens singulier à ce qui relève d'un patrimoine populaire commun. Si ces pratiques semblent prendre toute leur force au sein de l'intimité familiale, cela n'empêche pas les mêmes interprètes de se produire avec beaucoup de plaisir et de satisfaction devant un large public non manouche. Le concept d'«artification » nous aidera ici à mieux comprendre comment peut fonctionner cette articulation entre intimité et exposition publique. 


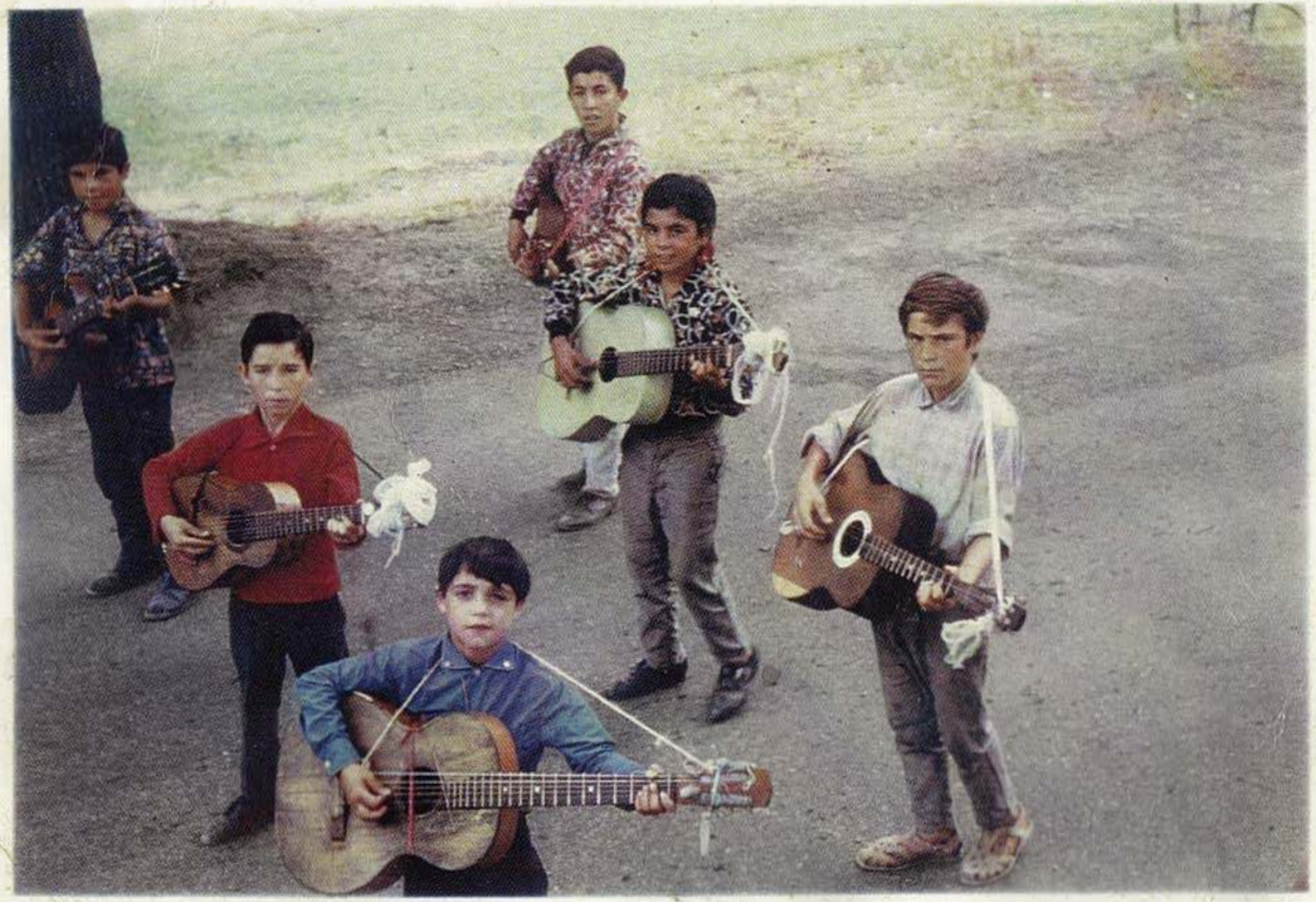

\section{ci-dessus}

fig. 1

Cinq jeunes Manouches jouant dans la rue à Marseille; à droite, le jeune Taylor, début des années 1960. La plupart d'entre eux se sont par la suite installés dans la région paloise. Avec l'aimable autorisation d'Éric Schumacher. (C) Photo D.R.
Les Manouches de Pau ne jouent pas de jazz manouche. Selon eux, cette musique n'est pas la leur. «Leur musique» repose pour l'essentiel sur des chansons françaises, anglaises ou espagnoles quelquefois, datant des années 1960 ou 1970 et dont l'interprétation en public rencontre un très vif succès auprès des nombreux Manouches locaux. Ils jouent aussi des instrumentaux aux consonances «classiques » ou d'innombrables bossas-novas, et chantent des airs d'opéra qui emplissent soudainement les petites cabanes bricolées dans lesquelles se déroulent les fêtes familiales. Ces productions ne prennent tout leur sens qu'au sein des familles, mais ces musiciens sont tout de même amenés, en diverses occasions, à jouer ces mêmes musiques «pour les gadjé»: ceux qui ne sont pas Manouches. Réfléchir à ce qui se joue dans cette oscillation entre intimité familiale et exposition publique revient à comprendre le sens particulier de ces pratiques musicales pour les familles, pour mesurer ensuite les enjeux d'une diffusion plus large.

II est question ici de Manouches catholiques de la région paloise, tous issus de groupes familiaux ayant voyagé en Espagne depuis le début du $x x^{e}$ siècle jusqu'à, pour certains, la fin des années 1950. Ces familles ont pu s'allier avec d'autres familles manouches venant de Marseille qui, elles, ne font pas de musique. Les familles pentecôtistes de la région paloise, originaires du Massif central, ont des pratiques musicales différentes qui nécessiteraient une étude à part entière. Nous n'en parlerons pas ici. 


\section{Jazz manouche et Manouches de Pau}

Les Manouches palois n'écoutent pas, dans leur très grande majorité, de jazz manouche. Ils n'évoquent pratiquement jamais Django Reinhardt sur le plan musical. En revanche, à Pau comme ailleurs, on trouvera toujours quelqu'un, généralement d'un certain âge, pour évoquer une anecdote à propos du fameux guitariste: Django était un lointain cousin, ou bien un « des plus grands buveurs » qui aient jamais existé, ou alors un autre, encore enfant, l'a vu jouer dans un café à Paris. Un jeune guitariste, qui ne joue jamais de jazz manouche, m'a dit regarder beaucoup de reportages et de sites internet consacrés à Django pour comprendre «comment un Manouche peut devenir une grande vedette internationale». Enfin, un musicien affirme régulièrement et avec beaucoup d'aplomb que Django n'était pas un Manouche. Quant aux autres styles musicaux attribués aux Tsiganes, qu'ils proviennent d'Espagne, des Balkans ou d'Europe centrale, les Manouches de Pau les ignorent totalement. Plus encore, ils désapprouvent clairement la manière très rauque, rancia, qu'ont les Gitans andalous de chanter le flamenco (Pasqualino 1994 et 1998).

Dans leurs propos, ils insistent sur ce qui les distingue des autres groupes tsiganes, y compris manouches, auxquels les gadjé auraient une fâcheuse tendance à les assimiler ${ }^{1}$. Quelles que soient les subdivisions raffinées que les gadjé élaborent concernant les classifications des Tsiganes (Tsiganes, Manouches, Sinté/Gashkéné manouches, Gashkéné manouches des Pyrénées...), celles-ci reposent toujours sur l'affirmation d'entités disposant d'une cohérence interne dont certaines manifestations culturelles pourraient attester. En revanche, pour un Manouche de Pau ou, du moins, quelqu'un se considérant comme tel, ce qui le lie aux autres relève plus du réseau que de la taxonomie: moi, mes enfants, mes parents, oncles, tantes, cousins et cousines proches ou éloignés, les ancêtres que je partage à des degrés divers avec eux, les relations que j'ai avec un certain nombre de gadjé de mon entourage, lesquels peuvent avoir été également liés à certains de mes «chers disparus", etc. Soit un système très souple d'alliances qui, en fonction de leur activation, entremêle petits groupes éphémères et familles nucléaires. Dès lors, naviguant entre le cadre familial et les interactions avec les «autres", les pratiques culturelles des Manouches de Pau ne se laissent guère cloisonner; elles se manifestent au contraire sous des formes multiples, selon les groupes qui se font et se défont au fil du temps, selon les événements et les liens nouveaux engendrés avec autrui (Williams 2011). Face à une telle complexité, ne reste plus qu'à tenter de décrire quelques manifestations musicales de cette diversité et à identifier, s'ils existent, les processus partagés qui les sous-tendent.

\section{Musiques écoutées et pratiques musicales ordinaires}

Les musiques qu'écoutent avec attention les Manouches que je connais sont presque toujours des musiques qu'ils pratiquent de manière active sous une forme ou une autre, que ce soit à travers le mouvement, le chant ou l'instrument.

Ainsi sur une aire d'accueil, en fin de journée par beau temps, on peut souvent voir des groupes d'enfants qui dansent près d'une voiture aux portes ouvertes dont l'autoradio diffuse de la musique de variété actuelle.
1. Observations déjà relevées par de nombreux anthropologues des Tsiganes (Williams 1993; Olivera 2012). 
Lors des fêtes, notamment à l'occasion des mariages, événements qui réunissent chaque fois plusieurs centaines de personnes, la musique oscille entre succès populaires récents et chansons françaises des années 1970 et 1980. Dalida suscite toujours un grand enthousiasme. Les gens dansent beaucoup, mais sagement. Une attention particulière est portée aux performances d'enfants qui s'affrontent dans des sortes de battles, garçon et fille, garçons entre eux ou filles entre elles, les adultes faisant cercle autour d'eux et les encourageant vivement. Dès le plus jeune âge, la musique s'incarne ainsi dans les corps. Toujours lors des mariages, certaines musiques accompagnent les moments solennels et en accentuent l'effet. C'est ainsi que souvent un morceau choisi pour l'occasion, grand air d'opéra, musique de film, etc., est diffusé lorsque le jeune couple qui s'était «enfui » arrive, vers onze heures du soir, dans la salle où tout le monde l'attend impatiemment, et remonte l'allée de papier crépon coloré qui mène jusqu'à l'estrade où sont juchés les parents.

Au sein des caravanes, dans les cabanes en bois qui servent de pièce commune à une famille nucléaire ou encore, et surtout, dans des fourgons appelés «camions » ou dans les voitures dotées d'autoradios aussi puissants que délabrés, s'accumulent souvent hors de leurs boîtes des CD de chanteurs et chanteuses de variétés, généralement passés de mode mais toujours dotés de "belles voix»: il y a vingt ans, on pouvait entendre Tom Jones ou Engelbert Humperdinck, mais aussi Ginette Reno, chanteuse canadienne encore très populaire au sein de certaines familles et dont les chansons continuent à être reprises par de jeunes chanteuses. C'est parce qu'«elle chante des chansons qui s'adressent directement à ceux qui les écoutent, comme si elle leur parlait", dit à son propos un jeune homme qui cite en exemple la chanson Ça pleure aussi, un homme, chanson que son père, aujourd'hui décédé, aimait beaucoup et que j'ai entendue récemment, reprise avec beaucoup d'émotion, par une jeune femme à l'occasion d'une fête pour la sortie de prison de son beau-frère.

Charles Aznavour est également très populaire au sein de ces familles, qui ne manquent pas de se rendre au Zénith de Pau quand l'artiste vient s'y produire. De nombreuses anecdotes circulent d'ailleurs sur la sympathie que l'artiste manifesterait à l'égard des Manouches. Là encore, des chansons telles que La Mamma ou Comme ils disent connaissent un vif succès auprès de chanteurs de plusieurs générations qui les reprennent a cappella à l'occasion d'une fête de famille ou les interprètent dans des bars à karaoké.

\section{ci-contre}

fig. 2

Moment musical entre membres d'une même famille dans la région paloise. (C) Photo Didier Sorbé.
Voici une quinzaine d'années que les familles que je connais aiment se rendre ensemble, maris, femmes et grands enfants, dans des bars à karaoké, chaque chanteur ou chanteuse ayant «sa " chanson, My Way pour l'un, une chanson de Johnny Hallyday pour un autre, Femmes, je vous aime pour un troisième... La prestation vocale a beau se répéter presque à l'identique depuis des années, l'interprétation de chaque chanteur ou chanteuse est toujours écoutée et applaudie avec beaucoup d'enthousiasme, comme si bien autre chose était alors salué.

De nouveaux chanteurs de variété internationale sont actuellement appréciés des jeunes générations mais leur style et parfois, nous le verrons, 


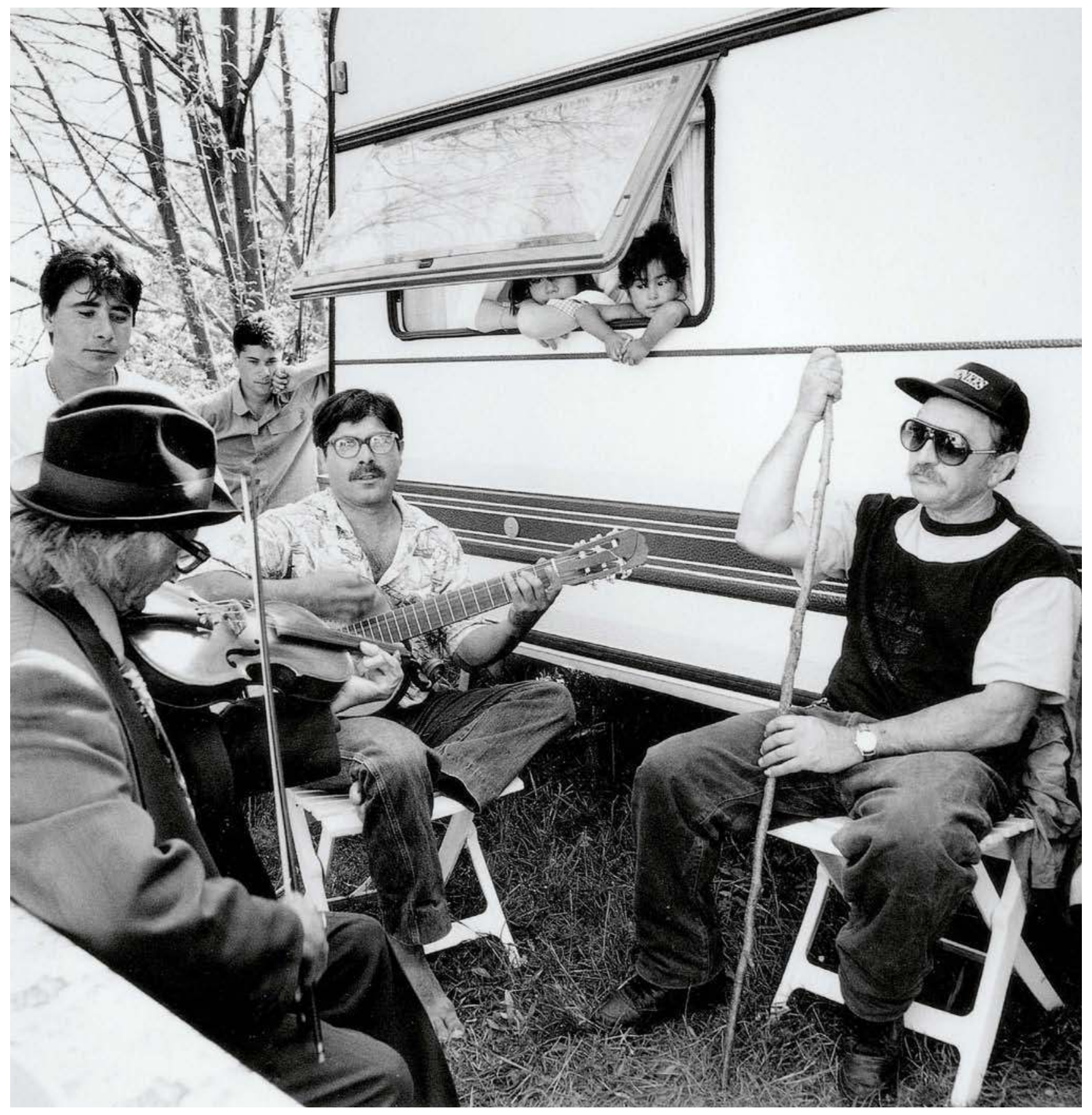




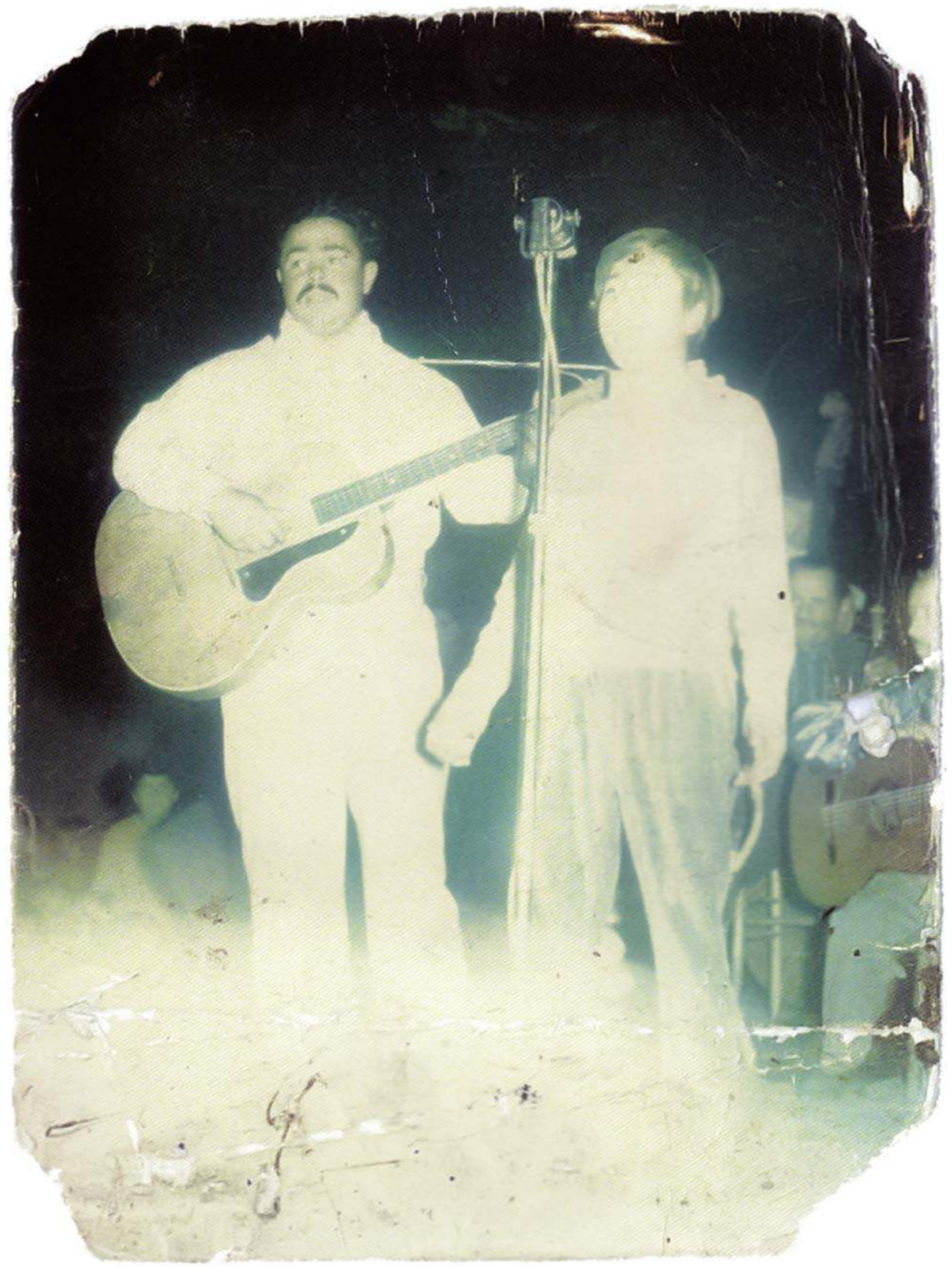


leur répertoire restent très sensiblement le même que celui de chanteurs plus anciens «à belle voix».

L'écoute de ces musiques a longtemps reposé sur celle de cassettes diffusées dans une voiture, remplacées depuis par des CD. Actuellement, pour les jeunes Manouches comme pour bien d'autres de leur génération, l'accès aux chansons se fait beaucoup par le biais des vidéos diffusées sur YouTube, à partir de smartphones. Parfois, une somme d'argent conséquente peut être consacrée à un déplacement pour un concert. C'est ainsi qu'à l'occasion du concert du chanteur mexicain Luis Miguel en mai 2012, trois jeunes cousines ont fait le déplacement de Pau à Barcelone (six à sept heures de route) pour aller le voir chanter dans un stade dans lequel se trouvaient «beaucoup d'autres Manouches et des Gitans espagnols».

En revanche, très peu de références sont convoquées concernant la musique instrumentale, si ce n'est celle de Luis Salinas, guitariste argentin de jazz qui joue de la guitare électrique, mais également de la guitare acoustique à cordes de nylon. Ce manque d'intérêt est à mettre en corrélation avec le nombre restreint d'instrumentistes sur la place de Pau.

\section{L'appropriation}

Comme en bien d'autres domaines (la cuisine, l'habitat, la nomination...), le processus d'appropriation qui consiste à donner un sens particulier à ce qui est commun s'avère prédominant dans le cas des pratiques musicales des Manouches (Piasere 1994; Poueyto 2012). C'est ainsi, par exemple, qu'une chanteuse âgée d'une soixantaine d'années, vivant à la campagne et qui se produit souvent avec son mari à l'occasion de petites fêtes de gadjé telles que des repas de chasseurs ou des anniversaires de mariage, m'a demandé si je pouvais lui trouver les paroles de la chanson Au premier rang de Ginette Reno parce qu'elle voulait la chanter «pour nous! Pas pour les gadjé». Intrigué par ce choix, j'ai constaté qu'il s'agissait d'une chanson parlant de parents disparus. Outre qu'une évocation discrète de leurs morts est une thématique omniprésente chez les Manouches (Williams 1993), on remarque ici un phénomène d'appropriation d'une chanson (qui n'est pas manouche) pour la transposer dans le cadre de l'intimité familiale: cette même personne m'avait déjà sollicité auparavant pour que je lui transcrive le texte de la chanson Les Vieux Mariés de Michel Sardou afin qu'elle puisse la chanter au mariage de son plus jeune fils ${ }^{2}$.

D'autres chansons, transmises de génération en génération depuis près de cinquante ans au sein de certains groupes familiaux, ne cessent d'émouvoir, voire de bouleverser, tous ceux qui, à des moments exceptionnels, seuls ou de préférence en groupe, les écoutent attentivement. Si certaines d'entre elles, chantées en manouche, peuvent être considérées comme “traditionnelles ${ }^{3}$ ", d'autres sont apparemment banales, leurs textes souvent anodins ou peu compréhensibles parce que chantés dans une langue étrangère plus ou moins maîtrisée (espagnol, italien, anglais), leurs rythmes et leur couleur sonore désuets.

\section{L'Espagne et Marseille}

Joseph Doerr, dit «Coucou », est connu pour avoir peint des tableaux
2. Qu'elle me demande un texte écrit m'étonne à chaque fois car je sais qu'étant très peu lettrée, elle lit difficilement.

3. Telles Tchavo ou Blumeli, blumela, dans le CD Latcho Dives.

\section{ci-contre}

fig. 3

Taylor, chanteur et musicien manouche de Pau (1950-1996) se produisant enfant sur une scène dans la banlieue de Marseille, 1961. Avec l'aimable autorisation d'Éric Schumacher. (c) Photo D.R. 
4. Le lap est le nom intime de chaque Manouche, utilisé à l'intérieur des familles pour le désigner. naïfs, avoir été un musicien virtuose et l'auteur d'une sorte d'autobiographie intitulée Où vas-tu, Manouche? (Doerr 1982; à propos de ce livre, voir Poueyto 2010). La lecture de cet ouvrage nous fournit bien des explications sur les parcours de ces familles au cours du xxe siècle même si la période de la Seconde Guerre mondiale est très peu évoquée. On y apprend que des familles Doerr, Adèle, Adolf, Lagrené, Weiss, Chaudy, Zigler, etc., sont parties en Espagne au début du xx siècle pour échapper à la guerre de 14-18. Elles ne vivaient pas toujours ensemble mais étaient amenées à se croiser, se faire et se défaire sur les territoires, terriblement austères, qu'étaient alors la Vieille-Castille, l'Aragon, la Navarre et parfois la Catalogne. Coucou situe le retour d'Espagne de sa famille durant la guerre civile et signale que presque tous les Manouches réfugiés d'Espagne se trouvaient à Marseille en 1939 (Doerr 1982: 101), ville qui, avec Lourdes, concentre alors toutes ces familles. C'est ainsi qu'une génération entière est née en Espagne dans les années 1930 et 1940, comme en attestent leurs laps ${ }^{4}$ à consonance espagnole, pour ensuite se retrouver enfants à Marseille, notamment au camp dit «du ruisseau Mirabeau ", longtemps présenté comme un bidonville. C'est dans cette ville, selon les souvenirs recueillis auprès de certains d'entre eux, qu'ils allaient chanter dès l'âge de 10 ans aux terrasses de café afin de gagner un petit peu d'argent. Une vieille photographie en couleur montre ainsi six jeunes garçons d'une dizaine d'années, chaussés de pauvres sandales, vêtus de chemises colorées de style "gitan » et marchant dans la rue avec, en bandoulière, des guitares auxquelles il manque des cordes (fig.1). Le fils de l'un d'entre eux les identifie: ce sont «leurs» chanteurs manouches, devenus palois au cours des années 1960.

L'un de ces enfants, Ramuncho, un neveu de Coucou Doerr, fut ainsi repéré à Marseille pour sa très belle voix par le patron d'un bar du Vieux-Port devant lequel ils avaient l'habitude de chanter. Celui-ci le mit en contact avec un imprésario et l'enfant enregistra, de 1962 à 1966 et pour la maison de disques Philips, plus de trente chansons. Parmi celles-ci, treize étaient en espagnol (Cuando calienta el sol, Llamame, Andalucía, Granada, Esperanza...), douze en français (La Mamma, Guitare bohème...) et une dizaine en italien (Torna e Surriento, II mio campanile, Se non ho la chitarra...), dont certaines produites pour le film italien II Figlio del circo (L'Enfant du cirque) de Sergio Grieco, dans lequel Ramuncho joue avec Pierre Mondy. Après que sa voix a mué, il a encore enregistré deux disques qui ne comprennent que des chansons en français (fig.7). II aurait ensuite accompagné dans leurs tournées des chanteurs comme David Alexandre Winter, Michel Sardou et même Johnny Hallyday... Au cours des années 1970, il a préféré rejoindre sa famille installée à Pau, abandonnant ainsi sa carrière musicale. Il est mort dans un accident de voiture au début des années 1980.

On sait que, durant les années 1960 et 1970, de nombreux succès étaient repris dans diverses langues, soit par les créateurs de ces chansons eux-mêmes, soit par des artistes nationaux. Les chansons françaises mises à part, Ramuncho chantait dans des langues qu'il ignorait. Né à Marseille, il n'avait jamais appris l'espagnol et très peu voyagé en Italie. Selon ses proches, l'enfant avait cependant une étonnante capacité d'apprentissage. Mémorisant d'oreille les chansons que ses imprésarios lui proposaient, il pouvait ensuite les restituer en l'espace d'une journée dans une langue 
dont il ignorait tout au départ. C'est ainsi qu'il reprend la chanson Granada, un succès de Joselito, l'enfant prodige espagnol des années 1950 qui servit certainement de modèle pour les imprésarios du jeune Manouche. Bien que Ramuncho ait disparu depuis plus de trente ans, certaines de ces chansons sont encore reprises de nos jours par ses proches ou leurs descendants dès qu'une occasion s'y prête: baptêmes, fêtes de Noël et du Nouvel An, etc. II s'agit pour l'essentiel de chansons en espagnol, celles en français d'«après la mue» étant dédaignées, voire critiquées. Selon des commentaires entendus, le jeune chanteur avait été poussé par ses directeurs artistiques à interpréter ces chansons jugées sans grande valeur ${ }^{5}$.

La chanson Esperanza, tango créé en 1961 par Nino de Murcia, est régulièrement réinterprétée par $M$., I'un des frères de Ramuncho, de sa voix puissante de baryton. Cuando calienta el sol est une chanson très connue, écrite semble-t-il par Trini Lopez mais surtout popularisée par Los Machucambos puis reprise par Arielle Dombasle et enfin par Luis Miguel. Avant de savoir que Ramuncho l'avait enregistrée, je l'avais souvent entendue reprise par T., un Manouche que j'ai bien connu et qui faisait partie des enfants identifiés sur la photographie. Cet homme, qui chantait beaucoup dans les bars en échange d'un coup à boire, interprétait cette chanson à la fois pour son propre plaisir et celui de son auditoire, et en hommage à son cousin et ami décédé. Disparu lui-même il y a quelques années, il était le père d'une des jeunes filles parties à Barcelone pour écouter Luis Miguel chanter, «à cause de mon père», m'a-t-elle dit. Chacune des ces chansons peut être alors considérée comme mulli, c'est-à-dire liée de manière intime à un cher disparu6 6 - pour l'essentiel Ramuncho et, par la suite, tous ceux qui se sont approprié ses chansons. Mais celles-ci demeurent des succès populaires que l'on a plaisir à chanter devant un public d'«étrangers» tels que les clients d'un bar, le public gadjo d'une petite fête, etc.

Enfin, si certaines d'entre elles ont été accaparées plus particulièrement par tel ou tel chanteur ou chanteuse, il en est une que la plupart d'entre eux reprennent en privé ou en public, en l'intitulant le plus souvent Les Enfants de Bohème. Il s'agit là encore d'un enregistrement de Ramuncho dont le véritable titre est Guitare bohème ${ }^{7}$. II se peut que cette chanson composée par Marcel Dubel, qui était chef d'orchestre à l'Alcazar de Marseille, music-hall célèbre alors pour ses spectacles d'opérettes, et dont je n'ai identifié aucune autre version dans le commerce, ait été composée spécialement pour le jeune Manouche, ce qui pourrait expliquer le succès qu'elle rencontre dans la communauté paloise ${ }^{8}$.

\section{L'opéra}

Le goût marqué de la plupart des Manouches de Pau pour les chansons interprétées par des voix puissantes et qui semble trouver son origine à Marseille est à mettre en relation avec le grand intérêt que la plupart des membres de ces familles éprouvent pour l'opéra. Ainsi, il y a plus de vingt ans, j'avais remarqué un homme d'une soixantaine d'années qui passait une bonne partie de ses journées debout près de sa voiture à écouter cette musique. Interrogé, il m'avait parlé de sa passion pour l'opéra et de grands ténors tels que Luciano Pavarotti. II avait lui-même selon ses proches la réputation d'avoir été, jeune, un très bon chanteur. Cet homme avait vécu
5. II s'agit des chansons Le Cavalier, Saint François d'Assise, El Dorado, Le Printemps sur la colline (juin 1965) et Tu es mon premier amour (Nancy), J'ai vu pleurer ma mère, Combien d'heures, Chez toi (juin 1966).

6. Un objet mullo (f. mulli, pl. mulle) est un objet lié à un mort (Williams 1993: 6-7).

7. Guitare bohème se trouve sur l'album Ramuncho chante en français de 1963.

8. Le guitariste Moreno, que j'avais sollicité comme conseiller artistique et guitariste pour l'enregistrement du disque Latcho Dives, à la fin des années 1990, avait alors découvert cette chanson et choisi de l'incorporer pour un temps dans son propre répertoire. 
9. Soit de manière phonétique, sans donner du sens aux paroles et en imitant plus ou moins les inflexions de la langue d'origine.

10. Cette attitude n'est pas propre aux pratiques musicales des Manouches de Pau, on la retrouve dans bien d'autres musiques populaires telles que le cante jondo ou le blues rural d'avant-guerre.

11. "L'essence même de la voix, écrit Junzo Kawada est de disparaître sans laisser de trace à l'instant précis où elle cesse d'être émise. C'est pourquoi son émission peut revêtir le caractère d'un événement. Le langage est avant tout vocal et c'est en effaçant la voix, le souffle du langage, que l'écriture a pu se fixer dans l'espace et s'approprier la continuité. " (Kawada 1998: 11) en Espagne avec Coucou et ses frères, ainsi qu'à Marseille et en Italie. Il semble que ce goût s'était forgé au cours de ses voyages ainsi qu'à travers une forte présence du bel canto dans la ville de Marseille, dans le répertoire de l'opéra ou de l'opérette. Cet intérêt, assez savant, s'est transmis par la suite à sa descendance comme j'ai pu l'entendre un soir de Noël 2011 où deux de ses fils et un de ses petits-fils s'étaient lancés dans un concours de bel canto à coups de petits extraits de chants d'opéra italien, chacun s'essayant à pousser la voix au maximum, sans tremblements ni altérations de justesse de la mélodie mais dans un italien très approximatif. Plus récemment, dans la cabane d'un autre de ses fils, à l'occasion du Nouvel An 2013, celui-ci, après avoir évoqué son père, a mis un CD de grands airs interprétés par le ténor italo-américain Mario Lanza pour lequel il éprouve une grande admiration, me parlant de l'influence de Caruso sur ce dernier tout en interrompant parfois ses propos en chantant d'une voix puissante et très juste par-dessus le disque diffusé. Discutant de ce goût pour l'opéra, il a également évoqué un «ancien » qui aurait chanté avec Pavarotti et qui intervenait autrefois régulièrement en direct dans une émission de radio à Marseille. Enfin, on peut entendre plus d'une fois sur certains terrains, lorsqu'un jeune se sent particulièrement bien, quelques notes d'opéra italien qui surgissent puissamment, telle une saeta dans une procession espagnole.

\section{La voix}

Le répertoire des musiques manouches décrit jusqu'à présent est donc avant tout composé de chants, qu'il s'agisse de grands airs d'opéra ou parfois d'opérette, de succès français des années 1960 et 1970, de chansons italiennes ou espagnoles, ou encore de standards américains tels que Just a Gigolo ou My Way. Si les paroles des chansons françaises reprises restent fidèles aux créations originales, il n'en est pas de même des chansons espagnoles ou italiennes et encore moins des chansons anglaises, ces dernières pouvant être chantées en "yaourt ${ }^{9}$ ", parfois devant un vaste public, sans que cela inquiète ni les chanteurs ni leurs proches. Cela fait ainsi plus de vingt ans que j'entends $M$. reprendre, de sa voix formidable, My Way à la manière de Frank Sinatra, le tout avec beaucoup d'émotion partagée par le public. Les paroles sont plus qu'approximatives car il ne parle pas du tout anglais. Des phonèmes manouches viennent compléter de mystérieux vocables à consonance anglo-saxonne. Pourquoi procéder ainsi, puisqu'il semblerait plus naturel que M. reprenne Comme d'habitude, la version originale de My Way dont il maitriserait parfaitement les paroles? Peut-être parce que Sinatra était un chanteur italo-américain qui s'inscrivait dans la tradition du bel canto, voix ronde, pleine, très puissante et ne tremblant pas, contraire en tout cela à celle de Claude François. Mais on peut également proposer d'autres hypothèses. L'une d'entre elles serait que ce qui compte le plus est l'émotion portée par la voix, qui doit prendre le dessus sur le logos quitte à brouiller volontairement ce dernier ${ }^{10}$. Le plaisir de chaque auditeur consiste alors à retrouver et à savourer cette voix «en performance » et son irréductible présence, partage intense entre le chanteur et son auditoire puisque le chant est amené à disparaître au moment même où il est émis ${ }^{\mathbf{1 1}}$. De plus, ainsi que le soulignait Roland Barthes, «la voix humaine est en effet le lieu privilégié (eidétique) de la différence: un lieu qui échappe à toute science, car il n'est aucune science (physiologie, histoire, esthétique, psychanalyse) qui épuise la voix» (Barthes 1982: 247). Pour les Manouches 


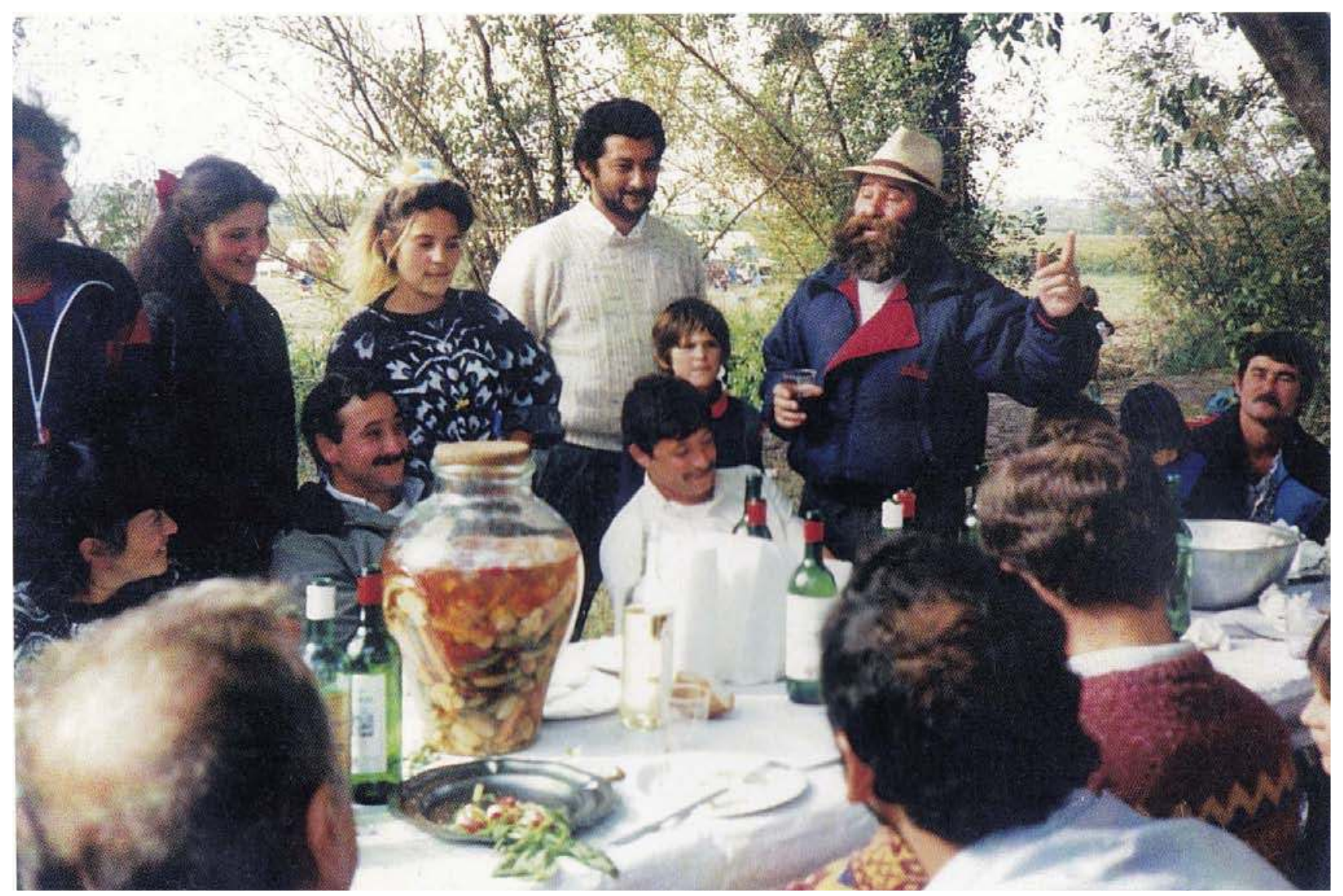

fig. 4

Taylor, parmi les siens lors d'un repas de vendanges dans les années 1990. II fut un chanteur et musicien très apprécié à Pau, aussi bien par les gadjé que par les Manouches. Avec l'aimable autorisation d'Éric Schumacher. (c) Photo D.R. 
12. Phénomène qui pourrait évoquer les chants des Roms de Hongrie décrits par Michael Stewart (1989), si ce n'est que ceux dont je parle ici relèvent toujours du répertoire gadjo.

13. Malheureusement introuvable.

14. II est à noter que de telles émotions ne se limitent pas aux familles elles-mêmes. J'ai pu moi-même partager au cours d'une soirée, en compagnie d'amis gadjé "respectueux", une écoute très émue de ces enregistrements qu'un ami manouche nous faisait écouter.

\section{ci-contre}

fig. 5

Sortie de l'église à l'occasion du pèlerinage manouche de Pardies Piétat, dans les Pyrénées Atlantiques. (C) Photo Didier Sorbé. comme pour bien d'autres chanteurs de musiques populaires, ce qui doit se communiquer est au-delà du logos: la présence de voix singulières qui s'inscrivent dans un héritage familial tissé, au fil du temps, avec des éléments piochés dans l'environnement culturel gadjo.

Chanter est avant tout une affaire de partage, de ce qui divise autant que de ce qui met en commun. En effet, le chant permet d'affirmer son unicité, chaque voix étant toujours singulière. Mais cela renforce aussi la cohésion du groupe: mêmes goûts, mêmes «manières " de lancer la voix, mêmes références à de "chers disparus" qui affirment l'existence d'un "nous", «nous" qui sommes parents et qui vivons et partageons tant d'expériences depuis tant de générations ${ }^{\mathbf{1 2}}$. Enfin, ce partage s'étend également aux autres puisque ces chansons sont également des chansons de gadjé et peuvent être interprétées dans des bars ou sur des scènes avec tout autant d'intensité et de plaisir, même si elles ne revêtent alors pas exactement le même sens.

\section{L'aura}

Il est actuellement assez facile de se procurer sur internet certains disques de Ramuncho. Les jeunes neveux ou petits-neveux du chanteur sont très au courant des sites qui s'y réfèrent, ne serait-ce que dans l'espoir de se procurer une version du film cité plus haut ${ }^{13}$. Ses 45 tours sont conservés au sein des caravanes, dans un sac ou un tiroir qui peut recueillir d'autres objets mulle, sans être jamais écoutés car personne ne possède de tourne-disque. En revanche, un certain nombre de versions de ses chansons circulent chez ses proches et sont écoutées avec beaucoup de respect, la plupart du temps dans la cabine d'un "camion » à l'arrêt, c'est-à-dire dans un espace où l'on peut s'isoler, contrairement à celui de la caravane ou des cabanes en bois, sans cesse traversées par les enfants.

Ces enregistrements, réunis maintenant sur des disques compacts, ont été obtenus initialement à l'aide de petits magnétophones à cassette au cours de fêtes familiales qui ont eu lieu aussi bien avant qu'après la mort du chanteur. On peut y entendre Ramuncho lui-même mais également un certain nombre de ses frères, cousins et amis, ayant tous partagé une enfance commune en Espagne puis à Marseille avant de venir s'installer, jeunes adultes, à Pau. Sur ces CD, la version de La Mamma mise à part, ce ne sont pas des copies des disques de Ramuncho que l'on entend, mais des moments saisis où ce dernier et ses proches chantent au milieu de guitares plus ou moins accordées, d'un violon lointain, de bavardages en manouche divers et bruyants, d'entrechocs de verres... La force de ces enregistrements repose donc à la fois sur le choix des chansons interprétées, les voix des chanteurs et plus particulièrement celles des disparus, mais également sur ces instants de vie partagés et captés de manière brouillonne par le magnétophone ${ }^{14}$. C'est dans ces documents sonores, en cela bien différents des morceaux gravés pour Philips, que chacun peut retrouver ce que l'on pourrait appeler l'aura, cette «trame singulière d'espace et de temps, unique apparition d'un lointain, si proche soit-il », selon Walter Benjamin (2000).

\section{L'héritage classique des Doerr}

Dans son livre, Coucou Doerr évoque son père, fameux violoniste semble-t-il, ainsi que ses oncles, tous musiciens. Selon lui, «[ils] ne connais- 


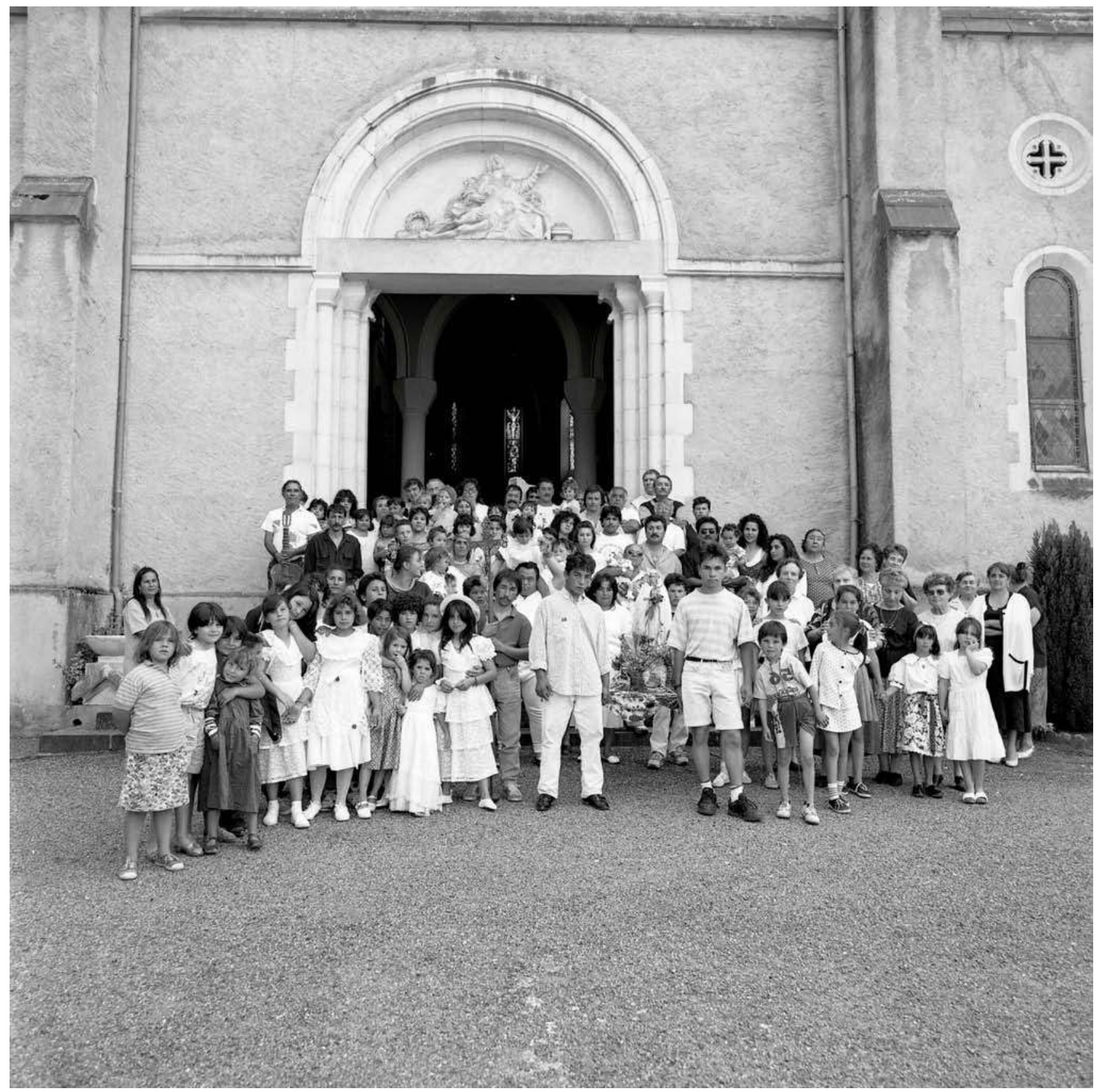


saient la musique que d'instinct et d'oreille mais pourtant ils jouaient de grandes symphonies comme Guillaume Tell, Poète et Paysan, de nombreuses sonates et de grandes valses " (Doerr 1982: 16). Puis il évoque une scène où il accompagne à la guitare son père qui interprète une polonaise de Pablo de Sarasate (Coucou écrit «Saradate»), violoniste espagnol de Pampelune qui vécut jusqu'en 1908. II décrit également une scène où Toumela, personnage important dans ce livre, un très vieil homme, improvise sur des thèmes de Niccolò Paganini (ibid. : 74). On peut se demander comment Lili, le père de Coucou, ses frères ou encore Toumela connaissaient ces morceaux relevant d'un genre savant puisqu'ils ne connaissaient la musique que “d'instinct et d'oreille». La scène décrite ici semble se dérouler à une époque où la radio n'existait pas encore (elle apparaît en 1920) et où seuls des rouleaux ou des disques de cire pouvaient permettre, si on possédait les appareils de diffusion adéquats, de prendre connaissance de telles musiques. Mais Coucou n'y fait nullement allusion. Et même si les morceaux évoqués ne sont que des ouvertures dont le thème au violon est relativement simple et lent (c'est du moins le cas de l'ouverture du Guillaume Tell de Gioachino Rossini ou de celle de Poète et paysan de Franz von Suppé), on peut supposer qu'un minimum d'écoute était nécessaire pour en retenir la mélodie et pouvoir la reproduire à l'identique. Quant aux interprétations de Paganini, elles devaient nécessiter, outre la connaissance des compositions, un minimum de connaissance des techniques et effets inventés par le maître, tel le jeu sur double corde, et dont la transmission se faisait également par l'écrit. Pourtant, Coucou note bien que «Toumela entame son récital sur double corde en ré et en sol, improvisant sur des thèmes de l'immortel Paganini ».

Lorsqu'on aborde ces problématiques d'apprentissage et de mémorisation avec divers membres de sa famille, ceux-ci proposent plusieurs réponses. La première est qu'il y aurait eu, dans bien des petites villes et villages du nord de l'Espagne, des orchestres qui jouaient ce répertoire en public, que ce soit sur des places ou dans des kiosques. Les musiciens manouches qui pouvaient donc écouter gratuitement de telles musiques devaient alors certainement rencontrer les musiciens «classiques» pour échanger avec eux, me précise-t-on avec insistance. Une autre explication, très souvent avancée et que j'ai déjà entendue sur bien d'autres sujets, est l'affirmation que les Manouches apprennent très vite tout ce qui les intéresse et donc que de telles mélodies pouvaient être immédiatement mémorisées par leurs auditeurs. On me cite alors comme exemple, outre le cas de Ramuncho évoqué plus haut, telle ou telle personne capable de rechanter intégralement une chanson qu'elle vient d'entendre à la télévision. Si de telles allégations semblent parfois exagérées, il est cependant très probable que la distance prise avec l'écrit chez les Manouches favorise grandement de telles compétences mémorielles, déjà observées par ailleurs parmi de nombreux musiciens non lettrés. Enfin, une dernière proposition, qui semble assez réaliste, est que les thèmes en question n'étaient jamais vraiment repris à l'identique, mais toujours réinterprétés avec de nombreuses variantes jusqu'à se transformer parfois en de nouvelles compositions. Toutes ces propositions qui évoquent la mobilisation d'un minimum de moyens pour l'obtention d'un maximum d'effets me semblent pertinentes: l'écoute et l'observation attentives de musiciens «savants» dans un lieu 
public gratuit, la mémorisation des éléments principaux de ces musiques (mélodie, tempo, arrangements possibles) et enfin leur réinterprétation affranchie de toute contrainte de répétition à l'identique (à ce propos, voir Détienne 1981: 81) constituent les étapes successives d'un processus d'appropriation qui, à son terme, débouche sur l'invention d'un morceau devenu désormais manouche.

Cette tradition "classique» persiste encore chez certains membres de la famille de Coucou comme dans la musique de son fils Jackie, musicien au touché sensible et aux accords savants que l'on peut entendre sur le disque Musiciens manouches en Béarn dans deux compositions dont l'une au moins semble héritée de son père ${ }^{15}$. Par ailleurs, un petit-neveu de Coucou, Nial Doya, est devenu un guitariste connu dans le Sud-Ouest qui joue régulièrement sur scène avec des musiciens gadjé. II est l'auteur d'un disque réalisé avec deux de ses cousins et deux musiciens gadjé ${ }^{16}$. Très doué, capable de jouer aussi bien de la rumba catalane avec ses cousins gitans ${ }^{17}$ que du jazz manouche, il s'en abstient cependant et affirme vouloir continuer à jouer et composer dans le sillage de ses aînés, les anciens Doerr. La musique de Nial est qualifiée, sur certains sites internet, de «musique traditionnelle du monde ". II est vrai que, oscillant entre bossa-nova et Isaac Albéniz ou encore Manuel de Falla, se référant tantôt à Jean-Sébastien Bach tantôt à Chet Baker, ses compositions aux accords très complexes joués en arpèges et aux harmonies recherchées restent tout à fait imprégnées de cet esprit de musique savante hérité de Coucou et de Jackie, bien difficile à catégoriser.

Son cousin Stessy, arrière-petit-neveu de Coucou, guitariste et très bon chanteur, anime avec un autre de ses cousins et trois musiciens gadjé un groupe nommé «Les Fils du vent». Ensemble, ils se produisent sur le territoire national et jouent leurs propres compositions dont les textes sont en manouche et le style relève souvent de la bossa-nova. Ce genre musical, importé du Brésil dans les années 1960, semble avoir vivement influencé les musiciens manouches des Pyrénées depuis plusieurs décennies. On le retrouve dans une surprenante version instrumentale de Comme d'habitude enregistrée en 1992 à Perpignan avec essentiellement des musiciens palois et tarbais ${ }^{18}$. "Mais comment veux-tu, avec de si belles mélodies, ne pas être inspiré!» me dit ainsi Stessy à propos des bossas-novas jouées dans sa famille, définissant ainsi un idéal musical Doerr fait de douceur et d'harmonie. Qu'il s'agisse de chansons passées de mode ou de compositions de type "classique", quel qu'en soit le rythme, tous ces morceaux, précise-t-il, reposent sur les mêmes types d'accords: des «accords Doerr». Se trouverait ainsi caractérisée une «manière Doerr» qui transcenderait tout style musical. Selon un musicologue local qui a transcrit un certain nombre de morceaux de Coucou, à qui je rendais compte de ces propos, il s'agit d'accords très courants en musique occidentale, la septième ou la quinte d'un accord majeur pouvant être par exemple jouées sur la corde de basse, comme dans l'harmonie classique, ou encore des accords de neuvième et onzième comme on en trouve dans les harmonies romantiques et jusqu'à Claude Debussy et Maurice Ravel. En revanche, sur le plan mélodique, l'intrusion de notes de passage étrangères aux gammes basiques provoque une impression de légère ambiguïté tonale qui donne une couleur particulière
15. Il s'agit de Voyage au bout de l'enfer et de Retour au pays, in Latcho Dives. À propos de Retour au pays comme support musical d'une mémoire familiale intime, voir Poueyto 2010.

16. Nial Doya et Tekenes, Pour Roman. Le nom du groupe était en fait "Ketenes", ce qui signifie "ensemble" en manouche, mais une erreur de frappe l'a ainsi transformé en un mystérieux vocable.

17. Une partie de sa famille est gitane, soit «espagnole» pour les Manouches.

18. Il s'agit du disque Zaitti. Musiciens manouches en Roussillon. 


\section{ci-contre}

fig. 6

Pilou Doerr, frère de

Coucou, se produisant lors du pèlerinage de Pardies

Piétat. () Photo Didier

Sorbé. à ces compositions. Enfin, une grande liberté rythmique rend ces morceaux souvent difficiles à transcrire dans les carrures habituelles.

Là encore, l'interprétation prenant le pas sur la reproduction à l'identique, il est fort possible que ce soit parce que les interprètes reproduisent d'oreille des morceaux entendus et y introduisent par tâtonnements successifs ou par associations d'idées de nombreuses variantes ou innovations; si bien que ces musiciens créent une musique qui se situe sans cesse dans un entre-deux bien difficile à classer, que l'on ne peut pas plus inscrire dans la catégorie «musique classique» que dans celle de «jazz manouche» ou même dans celle, si floue, de «musique tsigane». Seule l'étiquette «musique traditionnelle béarnaise" pourrait alors convenir, mais un tel classement risquerait de dérouter bien des amateurs de musique occitane.

\section{L'intimité et la publication}

L'inventaire commenté jusqu'ici évoque la transmission d'un répertoire de chansons et d'une manière de jouer la musique propres à un ensemble de familles partageant une longue expérience. Plusieurs générations de frères, de sœurs, de cousins et de cousines, nés les uns en Espagne, les autres à Marseille, les plus jeunes enfin à $\mathrm{Pau}$, ont vécu pour certains dans les campagnes arides et désolées d'Aragon, de Navarre ou de Castille pendant toute une partie du siècle. Ils se sont retrouvés à Marseille après-guerre, dans le même "camp pour nomades", sont partis dans la région paloise à partir des années 1960, ont partagé les mêmes matelas à l'arrière de vieux camions, les mêmes repas devant les caravanes, les mêmes grillades au bord des rivières, les mêmes déplacements pour effectuer des travaux saisonniers lointains, les mêmes pèlerinages, les mêmes mariages et les mêmes enterrements, et bien sûr les mêmes musiques populaires. Ces musiques font non seulement partie de leur répertoire commun de souvenirs, de partages multiples, intimes, mais de plus certaines chansons ont été enregistrées par des maisons de disques, sont régulièrement jouées dans des bars et restaurants bienveillants et même quelquefois sur des scènes très importantes. Ce jeu perpétuel entre intimité familiale et publication reste cependant d'une grande complexité, en renégociation permanente. C'est ainsi que, juste après la disparition de l'un de ses amis, un musicien était allé dès le lendemain jouer dans les restaurants. Comme bon nombre de ses proches s'en indignaient, s'attendant à ce qu'il marque le deuil par un arrêt momentané de son activité, il avait répondu que ce n'était pas la même chose puisque, pour les gadjé, il jouait «sans sentiment » et que cela ne comptait pas.

II serait cependant fallacieux de conclure que la "véritable musique manouche» se fait à l'intérieur des familles, conduite pour l'essentiel par le respect des morts, et que ce qui serait produit pour les gadjé serait secondaire, dénué de sens et uniquement motivé par des raisons pécuniaires. En effet, au-delà d'un répertoire partagé (chansons de variété, grands airs d'opéra, musique classique, bossa-nova...), la production de ces musiques est très étroitement liée aux rencontres incessantes entre musiciens manouches et non manouches. Les "anciens" allaient ainsi écouter les musiciens espagnols jouant de la musique classique ou qui chantaient de l'opéra à la radio. L'un d'eux fit sa carrière guidé par des imprésarios et accompagné par des orchestres professionnels. Les plus jeunes, enfin, 


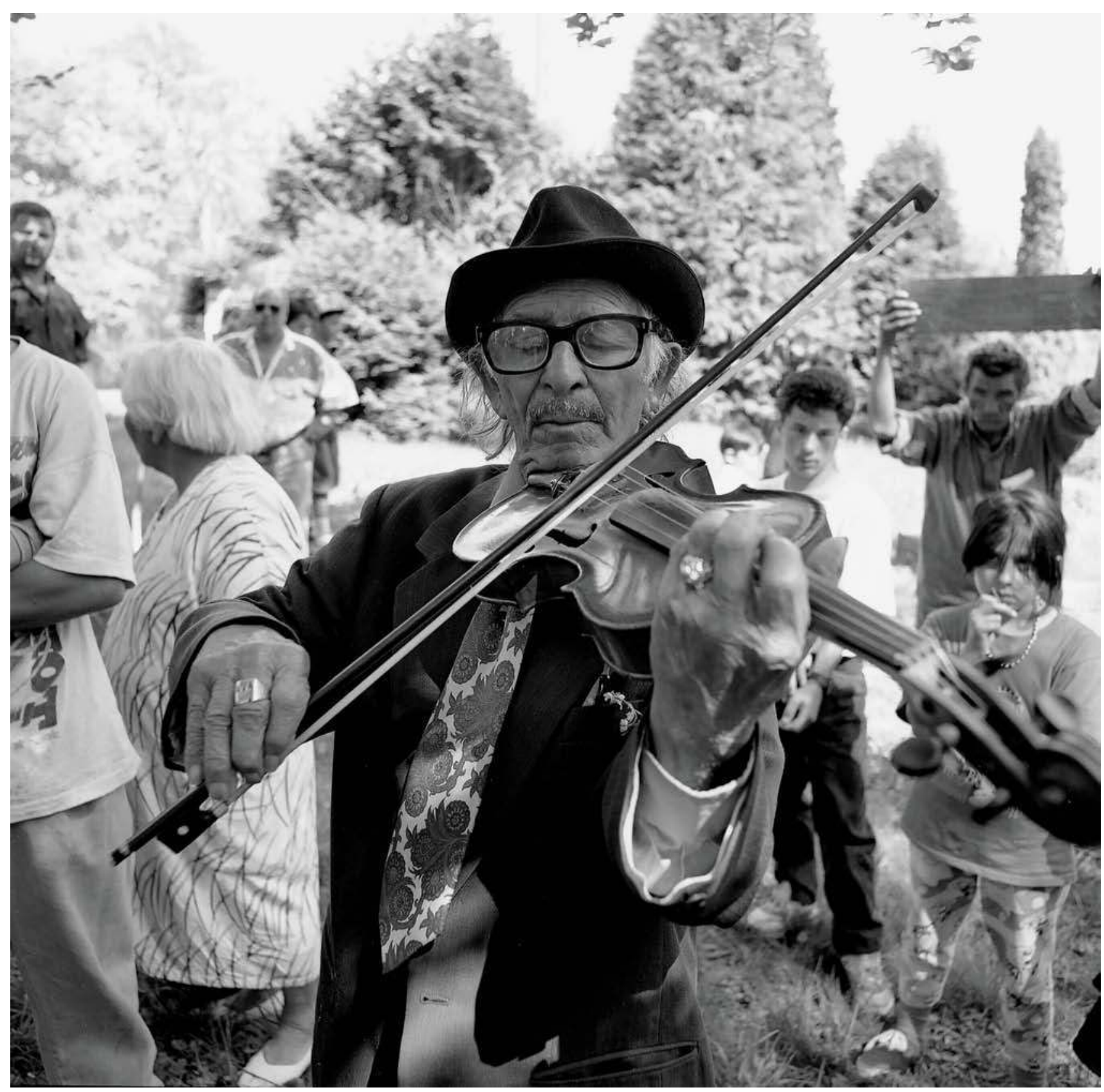




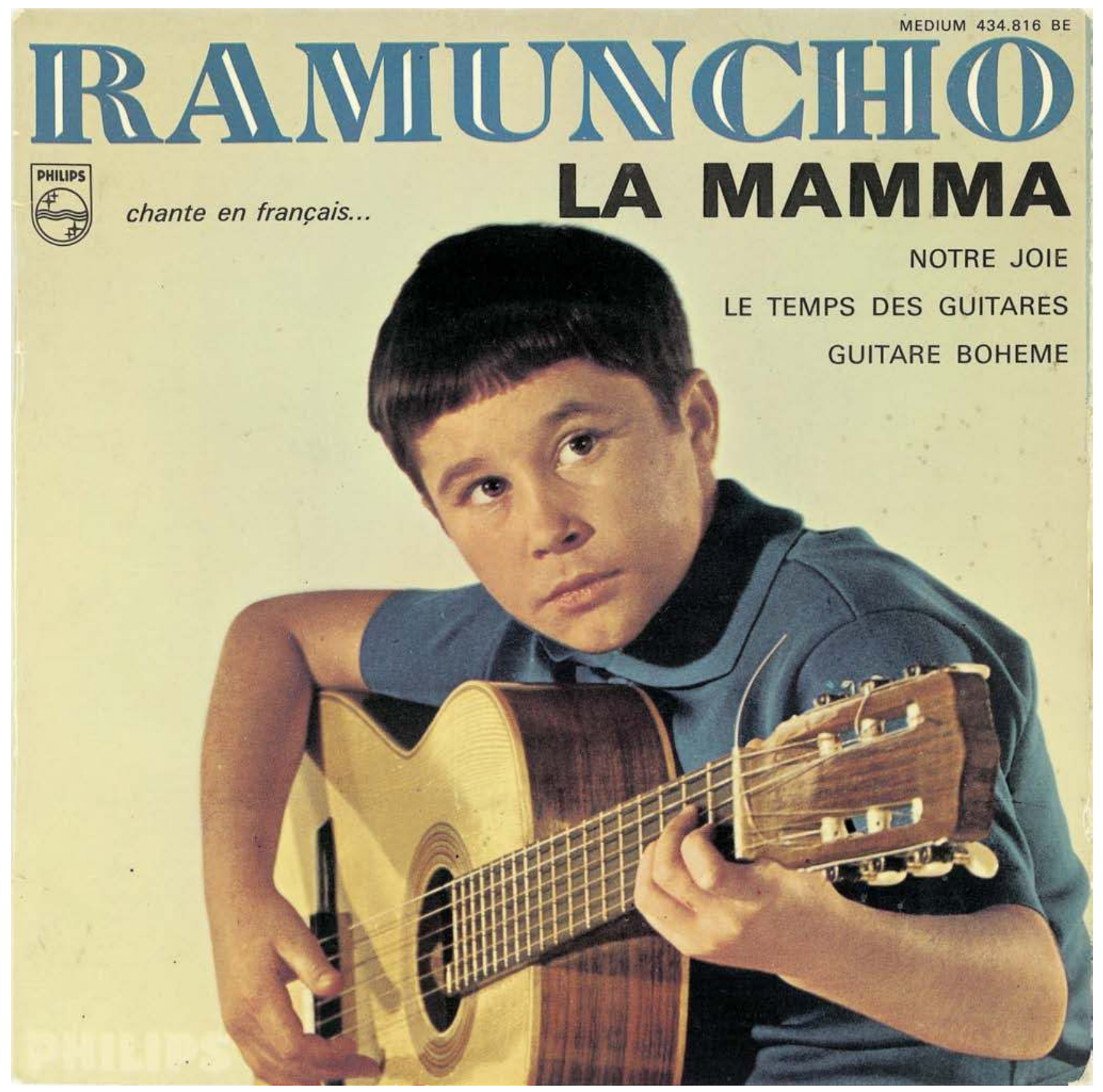


montent des groupes ou se produisent avec des musiciens gadjé, comme autrefois Django Reinhardt ou plus récemment Biréli Lagrène.

Ce mélange ne concerne pas seulement le cercle restreint des musiciens. En effet, j'ai pu observer combien, à différentes occasions, le public manouche de la région paloise pouvait être impliqué et ému à l'occasion de manifestations publiques organisées par des institutions. C'est ainsi que, en novembre 2011, une association locale intervenant auprès des gens du voyage ${ }^{\mathbf{1 9}}$ a organisé en partenariat avec une MJC paloise un événement intitulé «La semaine culturelle tsigane Latcho Dives Gadjé », avec conférences, exposition de tableaux dans un décor luxueux (le palais Beaumont de Pau) et grand spectacle musical dans un endroit tout aussi impressionnant, le Zénith de Pau. La mise en scène, réalisée par un des organisateurs, réunissait les musiciens manouches de Pau, bien sûr, mais également des écoles de danse locales, un ou deux rappeurs, un chœur d'une soixantaine de chanteurs et l'orchestre de Pau et des pays du Béarn (OPPB), grande formation très prestigieuse au niveau local. L'ensemble des manifestations fut activement soutenu par les institutions politiques. Un travail préparatoire conséquent avait eu lieu et les musiciens manouches avaient plusieurs fois répété avec les gadjé, qu'il s'agisse de rappeurs ou de l'orchestre classique. Tous étaient payés.

La soirée du spectacle a réuni plusieurs milliers de personnes, dont une grande majorité de gadjé, même si les Manouches de Pau étaient venus très nombreux. Sur cette très grande scène, les musiciens se sont succédé, jouant tantôt, pour les plus âgés, des instrumentaux qu'ils ont l'habitude d'interpréter ou, pour les plus jeunes, leurs propres chansons. Par ailleurs, le metteur en scène leur avait proposé un certain nombre de morceaux qui n'appartenaient pas à leur répertoire. De nombreuses collaborations ont vu le jour, le plus souvent sous forme de duos avec des musiciens gadjé tels un violoniste classique, des chanteurs de rap ou des DJ, un pianiste de jazz, etc., le tout entrecoupé de grands thèmes classiques évoquant les Tsiganes que jouait l'OPPB. Parmi les morceaux proposés, il y eut ainsi plusieurs versions des Yeux noirs, standard «tsigane » que les Manouches de Pau ne jouent jamais pour eux, et une reprise d'Ederlezi ${ }^{20}$, thème interprété par Goran Bregovic pour le film Le Temps des Gitans d'Emir Kusturica. Cette chanson fut interprétée d'une très belle voix et avec beaucoup d'assurance par une jeune fille de la famille Doerr accompagnée par le chœur et le grand orchestre classique. Plus tard, comme je questionnais son père sur l'origine de cette chanson, celui-ci me dit qu'il ne la connaissait pas auparavant et que sa fille ne savait pas en quelle langue elle l'avait chantée, bien qu'il s'agisse d'un dialecte romanès des Balkans dont un grand nombre de vocables peuvent être très proches du dialecte manouche sur un plan linguistique, mais dont la prononciation diffère grandement. Un tel choix musical, si éloigné de leurs pratiques (choix du morceau, orchestration, langue), avait de quoi déconcerter les spectateurs manouches présents. Ce fut au contraire le passage le plus commenté et apprécié par la suite.

II se peut qu'une telle mise en scène établisse de fait une grande distance entre l'intimité manouche et le danger de son dévoilement. II se peut également qu'elle offre là une forme de légitimation très bien accueillie
19. II s'agit de l'association béarnaise Gadjé Voyageurs et de la maison des jeunes et de la culture des Fleurs.

20. Le titre de cette chanson évoque la fête de saint Georges qui, en Bulgarie et en Serbie, est célébrée à l'occasion du début du printemps.

\section{ci-contre}

fig. 7

Pochette du disque de Ramuncho, La Mamma, 1963, Philips. 
21. "On dira donc que l'artification, c'est la résultante de l'ensemble des opérations, pratiques et symboliques,

organisationnelles et discursives par lesquelles les acteurs s'accordent pour considérer un objet ou une activité comme de l'art. C'est un processus qui institutionnalise l'objet comme œuvre, la pratique comme art, les pratiquants comme artistes, les observateurs comme public, bref, qui tend à faire advenir un monde de l'art. » (Heinich et Shapiro 2012: 21) par les Manouches, vis-à-vis des gadjé bien sûr, mais probablement aussi vis-à-vis d'eux-mêmes. Certains d'entre eux m'ont ainsi fait part de leur enthousiasme devant «une grande culture manouche» dont ils ne soupçonnaient pas la dimension. En s'inspirant de la grille d'analyse proposée par Nathalie Heinich et Roberta Shapiro concernant le phénomène de l'artification ${ }^{\mathbf{2 1}}$, on peut décomposer les effets de légitimation de cet événement en cinq «opérateurs», pour reprendre la terminologie des auteures. Le premier est d'ordre juridique: tous ces morceaux ont dû être déclarés à la Sacem, même s'il est bien difficile de savoir quelles œuvres pourraient être de véritables créations et quels en seraient les auteurs. De plus, les musiciens ont signé des contrats pour leur prestation, ce qui contribue très largement à une reconnaissance identitaire de musiciens professionnels et donc à un changement occasionnel de statut. Le deuxième opérateur, cognitif, institue qu'il s'agissait là d'un art et d'une culture «tsiganes». Les acteurs de cette manifestation ont participé à l'élaboration d'une catégorie culturelle qui satisfait bien des institutions politiques. En effet, un tel amalgame entre la chanson interprétée dans une langue de l'Est inconnue de la chanteuse et les multiples versions des Yeux noirs contribue à élaborer, même à tort, une catégorie culturelle pour un peuple unique, transeuropéen, réunissant les Roms de Roumanie, les Manouches de France et les Gitans d'Andalousie dans la même nation exotique, déconnectée de tout attachement territorial, local ou national. Le troisième opérateur est celui de la spatialisation: la musique était ici jouée sur la plus grande scène de la région, devant un public nombreux composé en partie de Manouches mais aussi très largement de gadjé. Jouer dans un tel lieu, connu par ailleurs pour accueillir les grandes vedettes internationales du spectacle, aidait largement à valoriser les prestations de l'ensemble des Manouches concernés. Le quatrième opérateur est institutionnel, car tout ce spectacle avait été conçu et organisé par des institutions du monde social. La réussite d'une telle soirée, qui s'appuyait sur l'affirmation d'une «culture tsigane», était donc étroitement liée à la reconnaissance de leur travail (Heinich et Shapiro 2012: 171). La question de la médiatisation est également très importante puisqu'une large communication avait été faite sur cet événement: grandes affiches vues par l'ensemble des Palois sur les panneaux d'affichage de la ville, flyers, nombreux articles publiés dans la presse locale, etc. Enfin, le cinquième opérateur contribuant à ce phénomène d'artification est d'ordre esthétique: l'accompagnement de l'orchestre de Pau et des pays de l'Adour a pu contribuer à légitimer cette musique. De même, les troupes de danse aux chorégraphies très convenues ont permis d'intégrer celle-ci dans un cadre déjà identifié par les spectateurs, celui des shows télévisés ou de certains grands spectacles populaires.

En revanche, et contrairement à bien d'autres processus d'artification tel celui qui concerne le jazz manouche, cette soirée n'aura rien fait pour la reconnaissance commerciale de ces musiques puisque, à ma connaissance, aucune maison de disque ni aucun tourneur professionnel n'était présent pour signer ou engager ces artistes.

En conclusion, nous sommes confrontés, en matière de musique chez les Manouches de Pau, à une dialectique, voire à un paradoxe, qui réunit et oppose tout à la fois la mémoire intime des familles, très implicite, 
silencieuse, complice et touchant presque au sacré, et son dévoilement à travers son exposition à un grand public gadjo. Mais une telle intimité ne se dévoile pas. Elle apparaît déguisée, sous une forme toujours un peu exotique comme lors du spectacle du Zénith, une forme qui laisse croire au public gadjo qu'il perçoit là une vérité manouche et qui leur offre dans le même temps une image un peu stabilisée, positive, unifiée et rassurante de ce monde, ce qui permet aux Manouches de perdurer dans le paysage palois sans trop de désagréments. Mais il se peut que tout ceci ne suffise pas à déboucher sur une musique originale reconnue par le public gadjo. La reproduction de mêmes formes, de mêmes chansons, de mêmes suites d'accords peut servir de support très efficace à une mémoire interne. Mais, à la différence de Django Reinhardt pour l'invention de ce qu'on nommera par la suite le jazz manouche, ou de Camaron de la Isla concernant la reconnaissance mondiale du flamenco, qu'aucun de ces musiciens n'ait encore comblé le fossé entre le sentiment très puissant qui habite la musique qu'ils jouent entre eux et la recherche d'une forme musicale nouvelle risque de les confiner dans une reproduction du même qui peut leur peser de plus en plus. À moins que... 
Loin de Django, la musique des Manouches de Pau Par Jean-Luc Poueyto

\section{Piasere, Leonardo}

1994 «Approche dénotationniste ou approche connotationniste? Les terminologies des parentés tsiganes", Études tsiganes (nouvelle série) 4 ("Jeux, tours et manèges. Une ethnologie des Tsiganes»): 183-208.

\section{Poueyto, Jean-Luc}

2010 "Coucou Doerr, un écrivain naïf? ", Études tsiganes 37

("Littératures romani : construction ou réalité? ").

2012 «Un patrimoine culturel très discret: le cas des Manouches», Terrains 58.

\section{Stewart, Michael}

1989 "La fraternité dans le chant, l'expérience des Roms hongrois", in Patrick Williams (dir.), Tsiganes, Identité, Évolution. Paris, L'Harmattan.

\section{et Shapiro, Roberta}

2012 De l'artification, Enquêtes sur le passage à l'art. Paris, Éditions de l'École des hautes études en sciences sociales.

\section{Kawada, Junzo}

1998 La Voix. Étude d'ethnolinguistique comparative. Paris, Éditions de l'École des hautes études en sciences sociales: 11.

\section{Olivera, Martin}

2012 La Tradition de l'intégration, une ethnologie des Roms gabori dans les années 2000. Paris, Petra.

\section{Pasqualino, Caterina}

1994 "La voix, le souffle, une séance de chant flamenco chez les Gitans de Jerez de la Frontera ", Études tsiganes 2

1996 Dire le chant, Anthropologie sociale des Gitans de Jerez de la Frontera, thèse de doctorat, École des hautes études en sciences sociales.

1998 Dire le chant, les Gitans flamencos d'Andalousie. Paris, Éditions de la Maison des sciences de l'homme.

\section{Williams, Patrick}

1993 Nous on n'en parle pas, des vivants et des morts chez les Manouches. Paris, Éditions de la Maison des sciences de l'homme.

2011 "L'ethnologie des Tsiganes ", in Michael Stewart et Patrick Williams [dir.], Des Tsiganes en Europe. Paris, Éditions de la Maison des sciences de l'homme.
Discographie

\section{Collectif}

Latcho Dives. Musiciens manouches en Béarn, à compte d'auteur, AîA CD 9906.

Zaïti. Musiciens manouches en Roussillon, ALCD 107, AI Sur ; téléchargeable sur le site http://www.alsur.fr.

\section{Nial Doya et Tekenes}

Pour Roman, à compte d'auteur, 201204CM.

\section{Ramuncho}

1963 La Mamma, Le Temps des guitares, Guitare bohème, Notre joie. Ramuncho chante en français, Philips, 434.816 BE, 45 tours.

1965 Le Cavalier, Saint François d'Assise, El Dorado, Le Printemps sur la colline. Philips 437045 BE,I 45 tours.

1966 Tu es mon premier amour (Nancy), J'ai vu pleurer ma mère, Combien d'heures, Chez toi. Philips, 437.226 F, 45 tours. 


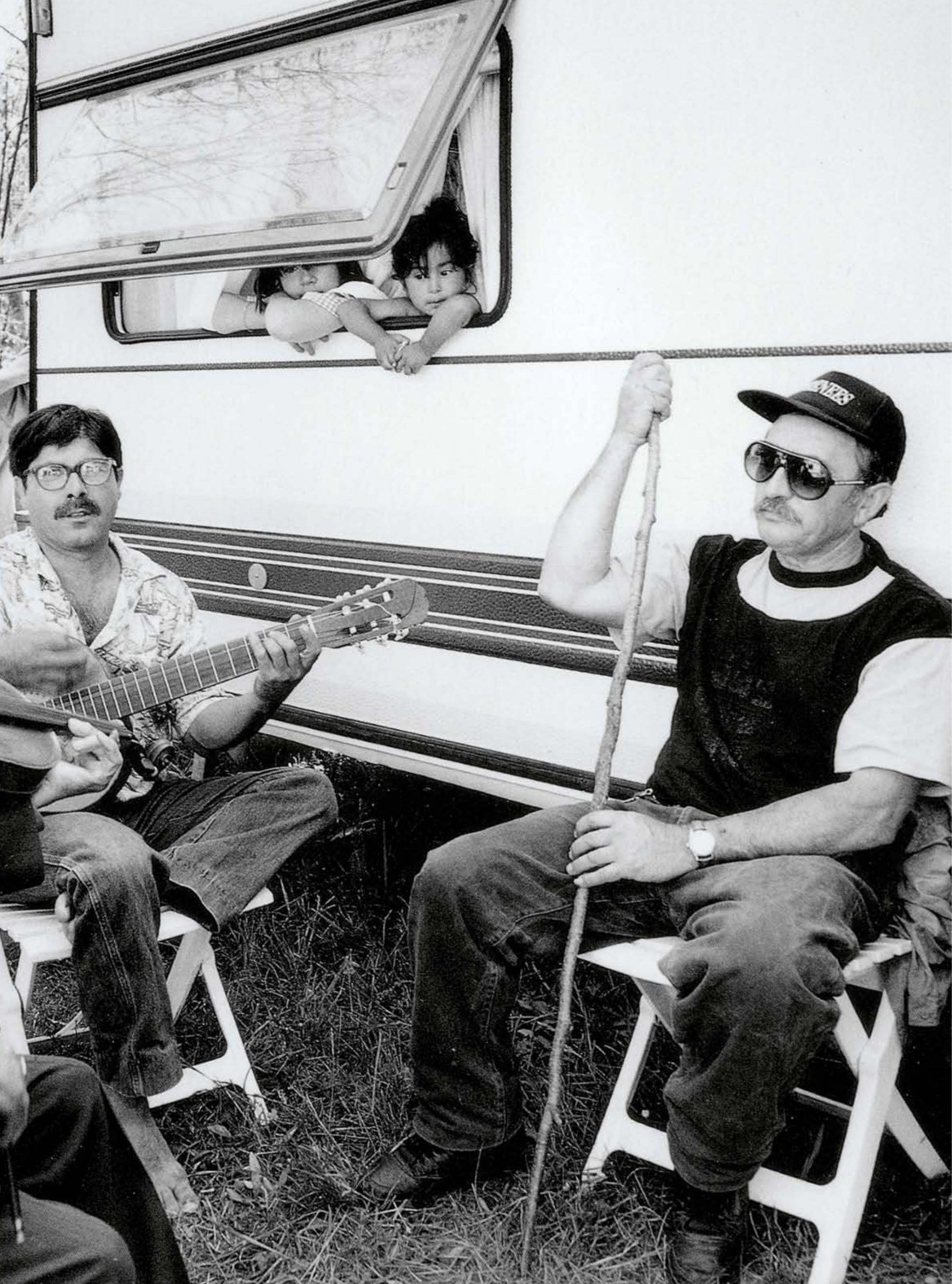

